\title{
Analysis of Water Features in Gas Leakage Area
}

\author{
Liu Huaishan, Wang Fengfan, Tong Siyou, Li Gaolin, and Zhang Haiyan \\ Key Lab of Submarine Geosciences and Prospecting Techniques, Ministry of Education, Ocean University of China, Qingdao 266100, \\ China
}

Correspondence should be addressed to Liu Huaishan, lhs@ouc.edu.cn and Li Gaolin, gaolin666li@163.com

Received 13 April 2011; Accepted 16 May 2011

Academic Editor: Xuewei Liu

Copyright () 2011 Liu Huaishan et al. This is an open access article distributed under the Creative Commons Attribution License, which permits unrestricted use, distribution, and reproduction in any medium, provided the original work is properly cited.

In a certain frequency range, gas is an effective absorber and scatterer of sound, which changes the compressibility of water, and then changes the speed and frequency of sound. Gas continues rising, deforming, and dissolving. The same bubble of natural gas has different radii at different depths. By analyzing these changes, the resonance frequency of gas bubble, and its impacts on sound wave, characteristics of the influences of gas at different depths on the incident sound wave can be obtained. The main sound features of gas are relevant to the gas size, gas content, velocity, attenuation, resonance frequency, the scattering cross-section, and so forth. Sound models with hydrate and free gas in the water and sediment are established. Through the practical application to actual data, the sound characteristics yielded when the gas (or gas hydrate dissociation) escaped the water of seismic data are very clear.

\section{Introduction}

There are various forms of marine gas, they may be generated by ocean waves, fish's swim bladder, submarine wake, shallow gas leak, hydrate decomposition, and so forth, they have a wide range of effects on sound signal used in marine seismic exploration, such as changing the speed of sound and attenuating signal energy. Various phenomena caused by natural gas have been found in the sea shallow gas and gas hydrates area, for example, speckled reflection "flame" shaped reflection, and so forth. These phenomena do not exist in an area without shallow gas and gas hydrates.

Carstensen and Foldy [1] proceeded with theoretical study of bubble scattering effects on sound propagation ignoring the relevance of the spatial distribution of bubbles. They obtained the bubble's radius by measuring the frequency of the sound generated by bubble, combined with the relationship between resonant frequency and bubble radius, and also obtained experimental data of the transmission and reflection coefficient of sound in the mixture. Experiments show that when the same bubble radius, and the incident wave frequency and the bubble resonance frequency are the same, the sound attenuates most, which is consistent with the theoretical value. Fox et al. [2] and others got the sound phase velocity and the sound absorption coefficient when the frequency is between $10 \sim 390 \mathrm{kHz}$ through incident continuous wave on the bubbly water. Keller and Kolodner incident sound with frequency $15 \sim 100 \mathrm{kHz}$ into the mixed-phase medium with the bubble (the radius of the $0.16-0.5 \mathrm{~mm}$ ), the result confirmed the Foldy theory once again. Silberman [3] measured the velocity and the attenuation coefficient of bubbly liquid in the static wave tube, the bubble radius is $0.08 \sim 0.26$ inches, and bubble volume fraction of 0.03 to $1.0 \%$, sound frequency is $60 \mathrm{~Hz} \sim$ $20 \mathrm{kHz}$. Xiaoliang et al. first proposed the concept of mixed media, obtained the sound wave equation in bubbly mixed media [4]. The researchers also found that the vibration characteristics of the bubble should be included in dynamic equations of mixed body. Prosperetti [5] linearized the vibration of air bubbles under the action of sound, studied heat conduction effects on the attenuation of sound, and found that the polytropic index and thermal conductivity were related to sound frequency. Kameda and Matsumoto [6] gave the mathematical model of nonlinear vibration of the bubble, and gave accurate description of the scope and characteristics of the effective equation. From his study we can find that Foldy theory and Van Wijingaarden equations are applicable in the case of lower volume fraction of 
bubbles that the interaction between bubbles can be ignored. Commamder [7, 8] and Prosperitti developed this theory based on Porsperetti's and others' accurate description of the dynamics on the bubble, and discussed the linear pressure wave propagation in bubbly water.

Li et al. [9] discussed the influences of the relevance of the spatial distribution of bubbles to sound propagation in bubbly water with consideration of Twersky's theory and sound absorption of bubble. The results show that when considering the relevance of the spatial distribution of bubbles, the attenuation coefficient of the peak moves backward and the peak is lower and flatter than ignoring the relevance. With the increasing of bubbles' volume fraction, the relevance of their spatial distribution increases, the resonant frequency of the bubble increases, and its scattering amplitude becomes smaller when resonating. Yonghui [10] used ultrasonic testing method to measure sound transmission loss and velocity ratio of the gas-water miscible media, the used sound frequencies were $0.5 \mathrm{MHz}$, 1.25 MHz, 2 MHz, and 2.5 MHz. Xiaoliang et al. [4] and others developed a series of analytical formulas that calculate the sound propagation characteristics in bubbly water and the nonlinear parameters of media for the fact that the actual bubble size always exists distribution. They studied the sound wave propagation features such as velocity and attenuation in bubbly water.

All the studies above are based on analysis of the impact extent of bubbles on the water, there are no studies on the sound characteristics of sediments and water containing hydrate and free gas in the gas leakage area. This paper studies the influence of gas on the effective sound absorber and scatter in a certain frequency range, natural gas radius at different depths when the gas continues to rise, deformation, or dissolved in the sea water. Then this paper analyzes that when these processes occur, how the resonance frequency of natural gas and the sound characteristics of it change. This paper established sound models with hydrate and free gas in the water and sediment through the analysis of gas leakage area (including hydrate and free gas) and real seismic data.

\section{The Rising Velocity and Track of Gas}

The rising of natural gas in water is a very complex process, and its rising velocity and track are related to the diameter and shape of natural gas. When calculating the rising process of natural gas, in order to simplify the calculation process, in theory, the diameter of natural gas is considered as the volumetric spherical to calculate, ignoring the internal activity and driven from setting velocity of solid particle in water.

The resultant force of natural gas in water equals to buoyancy force minus resistance force;

$$
F_{1}-F_{2}=\frac{\pi}{6} d^{3}\left(\rho_{w}-\rho_{a}\right) g-\frac{\pi}{2} C_{D} \rho_{w} \frac{v_{0}^{2} d^{2}}{4} .
$$

In $(1), d$ is the diameter of natural gas, $\rho_{w}$ is the density of water, $\rho_{a}$ is the density of air, $g$ is the acceleration of gravity, $C_{D}$ is resistance coefficient, and $v_{0}^{2} d^{2} / 4$ is the projected area of natural gas in vertical direction.

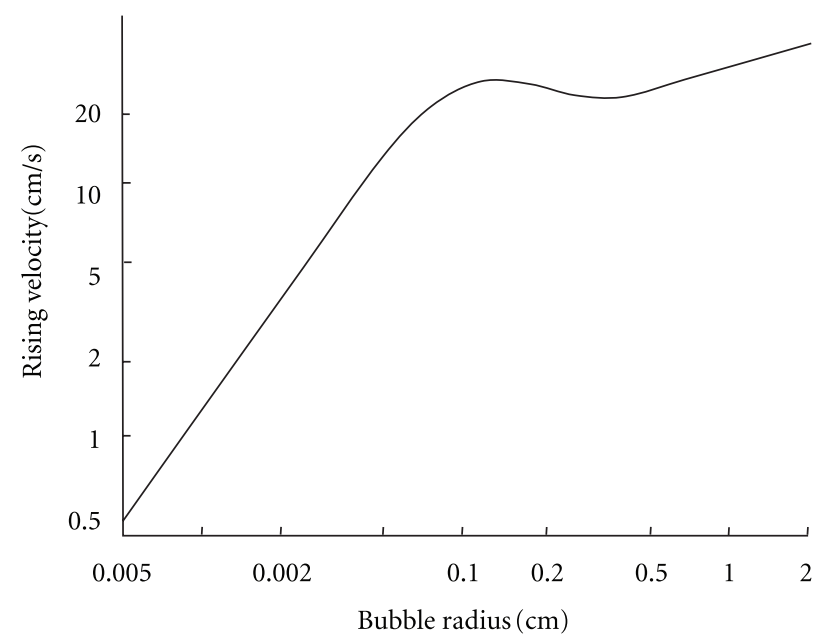

FIGURE 1: Curve between the rising velocity and diameter of natural gas.

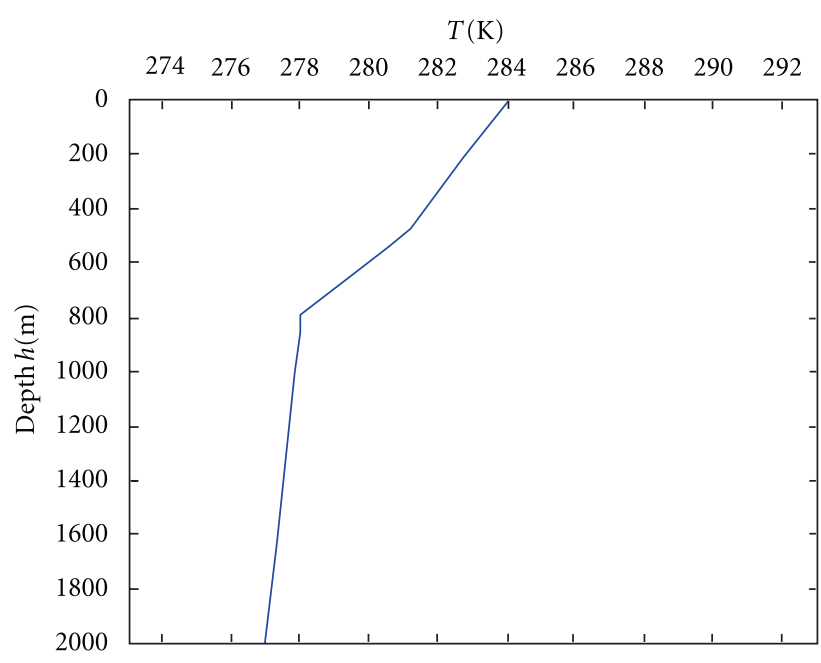

Figure 2: Curve of longitudinal temperature of seawater.

According to Newton's second law, we can know that $F=$ $m a, m=\pi d^{3} \rho_{a} / 6, a=d v_{a} / d t, v_{a}$ is the rising velocity of natural gas, if we put it into (1), we can get that

$$
\frac{d v_{a}}{d t}=\frac{\left(\rho_{w}-\rho_{a}\right) g}{\rho_{a}}-\frac{3 C_{D} \rho_{w} v_{a}^{2}}{4 \rho_{a} d}
$$

When $d v_{a} / d t=0, v_{a}$ is the ultimate velocity;

$$
v_{0}^{2}=\frac{4 g d\left(\rho_{w}-\rho_{a}\right)}{3 \rho_{w} C_{D}} .
$$

From hydromechanics knowledge, we can know that resistance coefficient $C_{D}$ can be determined by Reynolds number $R_{e}$;

$$
R_{e}=\frac{v_{a} d}{v}=\frac{\rho_{w} v_{a} d}{\mu} .
$$

In (4), $v$ is motion viscosity coefficient and $\mu$ is dynamic viscosity coefficient, respectively. According to the difference 


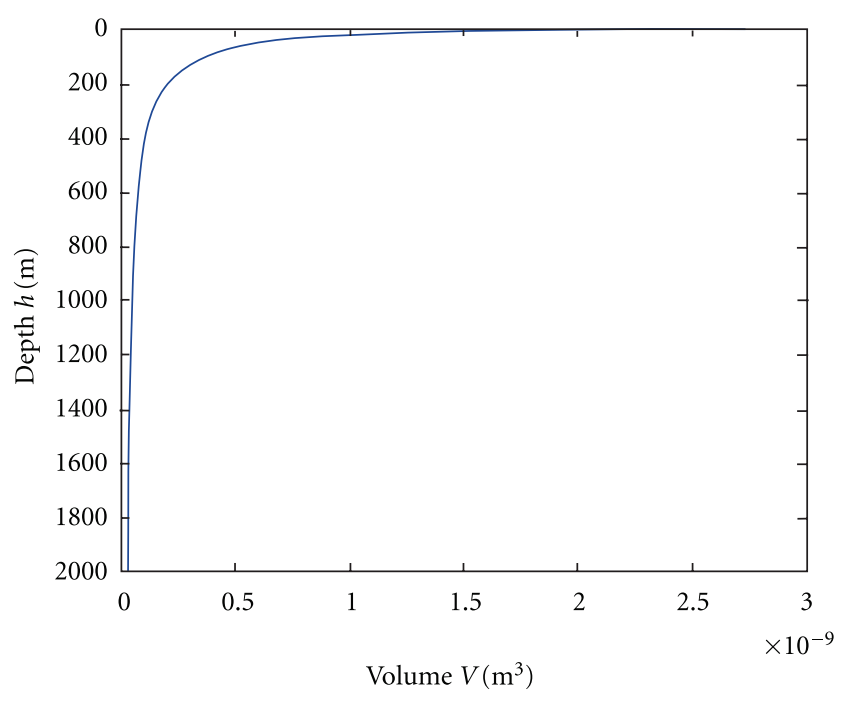

FIGURE 3: The relationship between the balance volume of natural gas and depth.

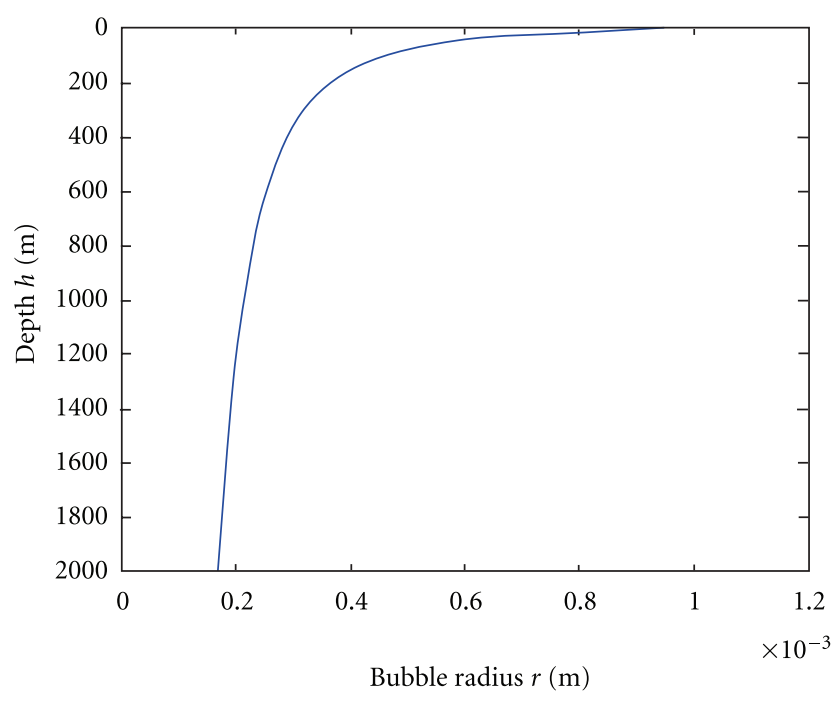

FIgURE 4: Curve of the balance radius of natural gas with depth.

of Reynolds number, the value of $C_{D}$ can be divided into three areas: laminar flow, transition, and turbulence. The velocity formula calculated from this is also different.

According to hypothetical conditions, (3) has a small range of application and usually is suitable to bubble whose diameter is less than $150 \mu \mathrm{m}$ in static water.

While in the rising process of moderate natural gas, there are two forms of motions [5]: (1) Rolling motion of rigid body, zigzag or spiral motion. (2) Changes in shapes and expanding, which is oscillation of natural gas. These forms of motion often exist at the same time, and movement of moderate natural gas is very complicated. But we can simplify its model to a perfect ellipsoidal and then consider its additional motions.

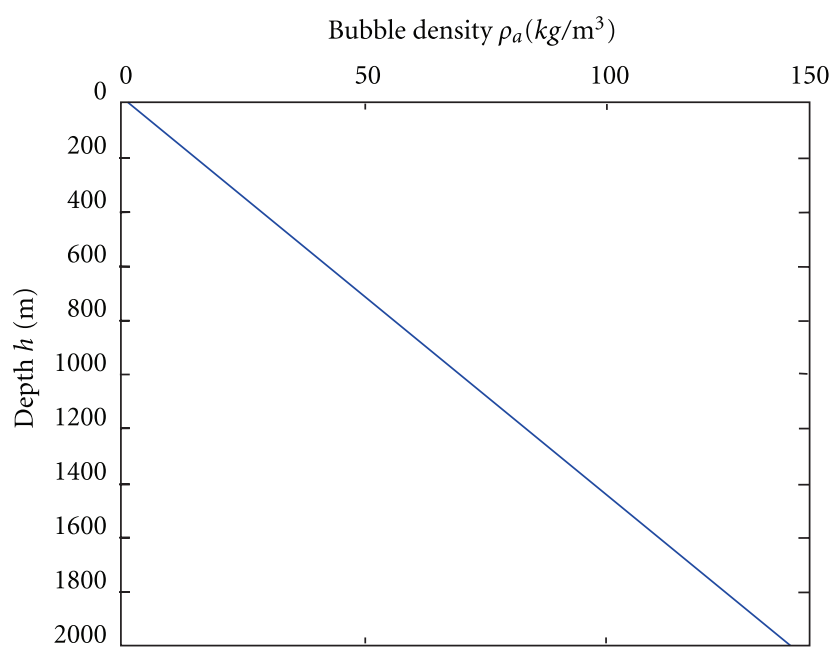

FIGURE 5: Curve of the density of natural gas with depth.

While with regard to big natural gas, its shape can only be acquired from observation and the expression of its velocity is considerably complex.

Therefore, the rising velocity of natural gas in static water is closely related to its linear density. According to huge amounts of experimental data and theoretical analysis, we can get the relation curve between the rising velocity and diameter of natural gas which is shown in Figure 1. It can be seen from Figure 1 that the velocity of natural gas reaches a maximum value when the diameter is about $0.1 \mathrm{~cm}$, and then changes very little with the increasing of diameter while the shape of natural gas changes from spherical to ellipsoidal. With regard to small natural gas, its characteristics of motion are linear. And for big natural gas, its characteristics of motion is approximate to linear, but its shape usually becomes distorted and not spherical any more. When the diameter is greater than $1000 \mu \mathrm{m}$, the motion of natural gas is not stable anymore and is not the function of diameter, so it is hard to calculate the velocity of natural gas. In experimental observation, its rising velocity is approximate to $30 \mathrm{~cm} / \mathrm{s}$.

In fact, in the rising process of natural gas, the volume will increase with the decrease of pressure and its shape will also change. The formation of water vapor in its interior changes the density of natural gas. And because of the existence of surface tension, the gas in natural gas will dissolve in the water. Experiments show that the natural gas whose diameter is $0.1 \mathrm{~cm}$ can completely dissolve in water within about $20 \mathrm{~min}$.

From the above analysis, we can see that the big natural gas in sea water will rise to the sea surface quickly, and tiny natural gas mainly exists in sea water. The minimum stable diameter in sea water is about $1 \mu \mathrm{m}$. Although this natural gas is still subjected to the influence of buoyancy force and gas diffusion to dissolve and rise constantly, amounts of measuring results in sea show that the densities of tiny natural gases in sea water will keep a dynamic balance state and diameters of natural gases are all less than $500 \mu \mathrm{m}$ [11]. 


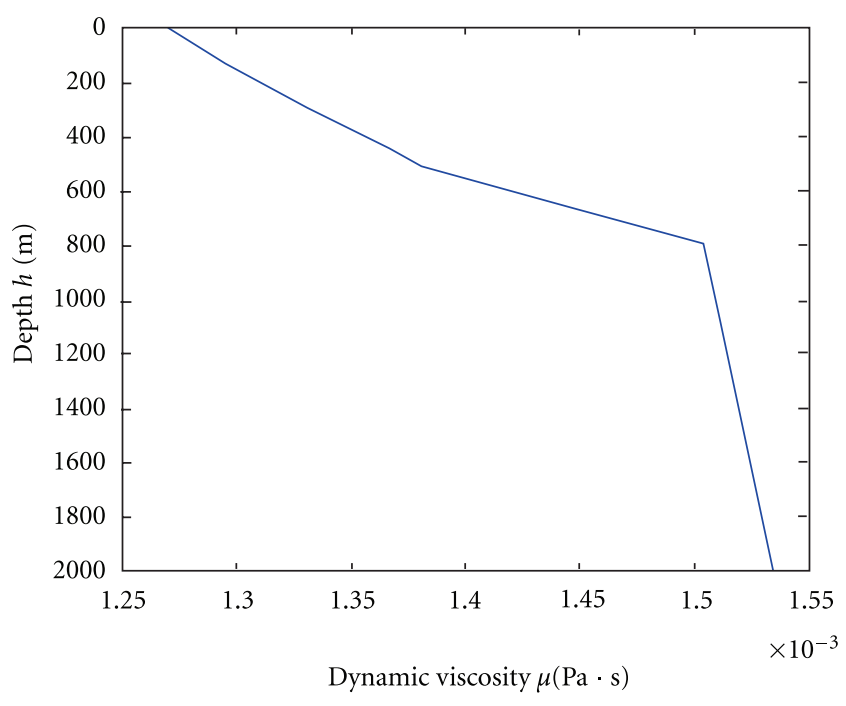

FIGURE 6: Curve of seawater viscosity coefficient with depth.

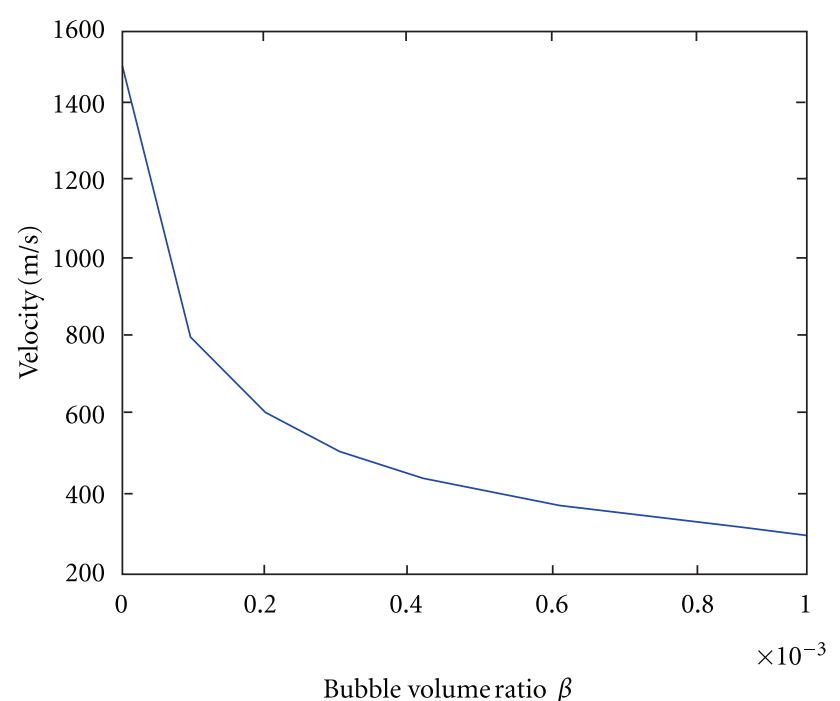

Figure 7: The curve of relation between sound velocity and the ratio of gas volume.

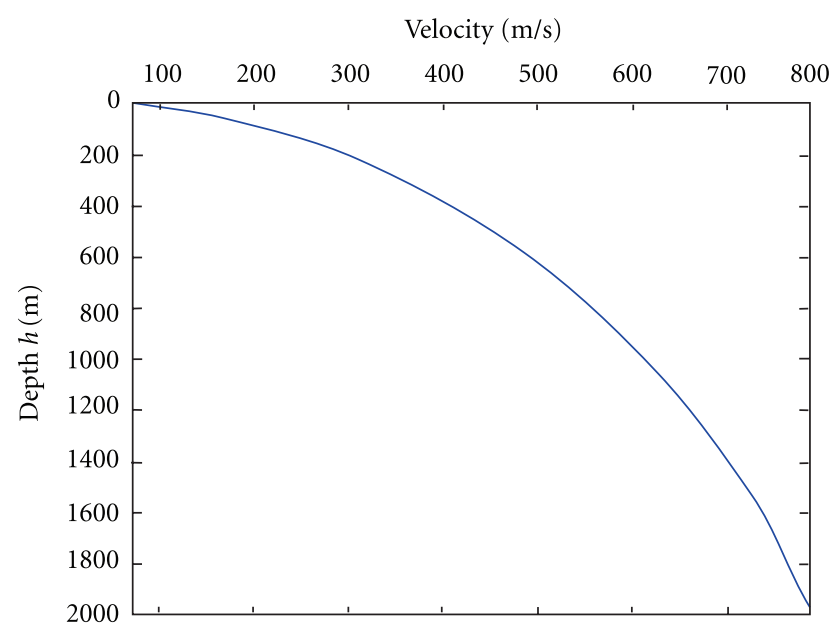

FIGURE 8: Sound velocity changing with depth in the gas layer.

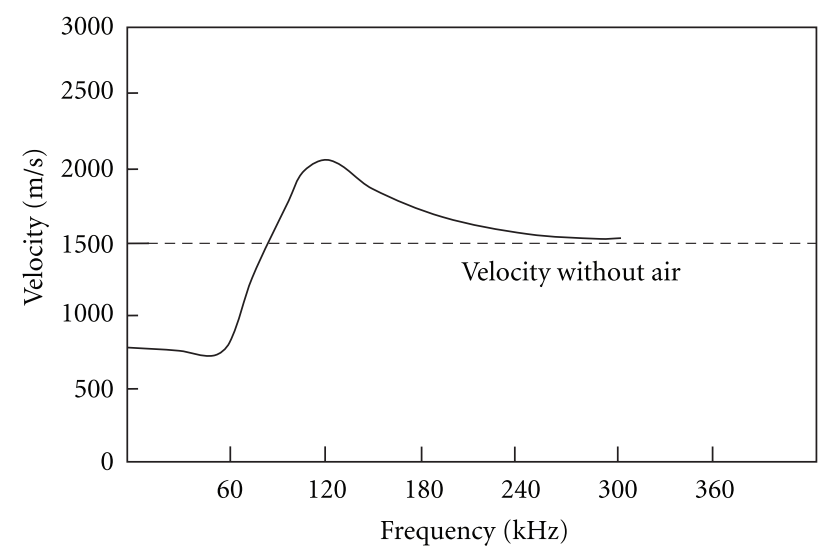

FIGURE 9: Curve of sound velocity measured in the gas of same size (diameter is $0.011 \mathrm{~cm}$ ) under wide frequency range [2].

2.1. The Rising Feature of Natural Gas in Water. Assuming that there is some natural gas in marine, its shape always remained spherical in the process of rising in static water, the radius in sea level is $1 \mathrm{~mm}$, and the volume is $V_{0}=$ $4.1888 \times 10^{-9} \mathrm{~m}^{3}$, which is full of methane gas.

The pressure in the sea level is one standard atmospheric pressure, and the seawater pressure in the depth of $h$ is $P_{1}=$ $P_{0}+\rho_{w} g h$. Suppose the seawater is incompressible fluid, $\rho_{w}=$ $1026 \mathrm{~kg} / \mathrm{m}^{3}$ is the average density of seawater, and the gravity acceleration is $g=9.81 \mathrm{~m} / \mathrm{s}^{2}$. The temperature in sea surface is $284 \mathrm{~K}$. The change of temperature is shown in Figure 2.

The molar mass of methane gas $M$ is $16 \mathrm{~g} / \mathrm{mol}$, according to the equation of state of ideal gas:

$$
\frac{P_{0} V_{0}}{T_{0}}=\frac{P_{1} V_{1}}{T_{1}}=n R,
$$


$R=8.31 \mathrm{~J} \cdot \mathrm{mol}^{-1} \cdot K^{-1}=0.082 \times 10^{-3} \mathrm{~m}^{3} \cdot \mathrm{atm} \cdot \mathrm{K}^{-1}$. $\mathrm{mol}^{-1}$.

We can obtain that the amount of substance of this natural gas is $n=1.7987 \times 10^{-7} \mathrm{~mol}$, its quality is $m=$ $n \times M=2.8779 \times 10^{-9} \mathrm{~kg}$, and the density in the surface is $\rho_{a}=m / V_{0}=0.68705 \mathrm{~kg} / \mathrm{m}^{3}$, then the relationship between volume of gas and depth can be obtained:

$$
V_{1}=\frac{P_{0} V_{0}}{P_{0}+\rho_{w} g h} \frac{T_{1}}{T_{0}} .
$$

In the equation, $T_{0}$ is the temperature in the sea surface, and $T_{1}$ is the temperature in the depth of $h$.

Assume that the internal gas composition and quality of the natural gas do not change in different depths of ocean water, then according (6) and the given data, we can obtain the change curve of gas volume with depth (Figure 3 ).

At the same time, the change of radius $r$ with depth $h$ can be found by formula $r=(3 V / 4 \pi)^{1 / 3}$ (Figure 4). As shown in the figure, at the beginning, the change of volume and radius of the bubble in the water depth of several hundred meters is very rapid, but along with the increase of depth, the change rates of volume and radius become smaller and smaller. According to the relationship between the quality $m$ of natural gas and volume $V$, the change curve of the density of natural gas can be worked out (Figure 5).

Without considering the composition change of natural gas, there is a linear relationship between the density of natural gas and depth:

$$
\rho_{a}=\frac{m T_{0}}{P_{0} V_{0} T_{1}}\left(\rho_{w} g h+P_{0}\right) .
$$

We can calculate that the volume of natural gas is $V=$ $2.05 \times 10^{-2} \mathrm{~mm}^{3}$ and the radius is $r=0.16966 \mathrm{~mm}$ in the depth of $2000 \mathrm{~m}$.

Thus we can assume that there is a methane gas with balance radius of $0.16966 \mathrm{~mm}$ in the depth of $2000 \mathrm{~m}$ below the sea surface, which rises freely in the case of static seawater.

Because the maximal radius of this natural gas is only $1 \mathrm{~mm}$, according to the actual observation data and calculation results, its maximal Reynolds number in seawater is far less than 1000 .

As known from the fluid mechanics, the object moves relative to the fluid in the fluid whose surface has a "boundary layer." The microregiment near objects in the layer is static relative to the object, and the fluid micro regiment on the outside of the layer has the same speed as fluid. So there are velocity grads and viscous force in the boundary layer, which can serve as resistance to the object.

According to the given conditions, the dynamic viscosity coefficients $\mu$ of different depths are calculated using water and steam properties (Figure 6).

Smaller objects move slowly in the fluid with large stickiness, which means, in the case of small Reynolds numbers, the resistance, called viscous resistance, is the main factor. The famous Stokes formula describes the viscous resistance of spherical objects: $F=6 \pi \mu v r, r$ is the sphere radius, $v$ is the velocity of sphere, and $\mu$ is viscosity. This formula is correct as the condition that Reynolds number is much smaller than 1 . That means when $\operatorname{Re} \leq 1$, it fits the conditions of Stokes resistance formula, $C_{D}=24 / \mathrm{Re}$, combined (4):

$$
\frac{d v_{a}}{d t}=\frac{\left(\rho_{w}-\rho_{a}\right) g}{\rho_{a}}-\frac{18 \mu v_{a}}{\rho_{a} d^{2}} .
$$

While $1 \leq \mathrm{Re} \leq 1000$, resistance coefficient $C_{D}$ is calculated by corrected White experience formula:

$$
C_{D}=\frac{24}{\mathrm{Re}}+\frac{6}{\sqrt{\mathrm{Re}}}+0.4 .
$$

Take formula (9) into formula (8):

$$
\frac{d v_{a}}{d t}=\frac{\left(\rho_{w}-\rho_{a}\right) g}{\rho_{a}}-\frac{3 \rho_{w} v_{a}^{2}}{4 \rho_{a} d}\left(\frac{24 \mu}{\rho_{w} v_{a} d}+6 \sqrt{\frac{\mu}{\rho_{w} v_{a} d}}+0.4\right) .
$$

Using formula (10) can calculate the transient acceleration of bubble in the process of rising, and making $d v_{a} / d t=$ 0 can obtain the final speed when the force is balance.

2.2. The Velocity of Natural Gas in Water. It is measured that the air dissolved in fluid (liquid) has no influence on the velocity of sound, even the air in the liquid is saturated. But if there is small gas saturated suspended in the water, even very little, it may decrease the sound velocity. The sound velocity is related to the proportion of the gas in liquid and the frequency. Because the gas has a better compression than water, the compressibility of the water will change as the increasing of the proportion of the air. According to the formula $c=\sqrt{1 / \rho_{0} \beta_{s}}\left(\beta_{s}=-(d V / V) / d P\right.$ is the adiabatic volume compressibility), the sound velocity changes apparently.

When the air's volume is smaller than the resonance bubbles, that means the frequency of sound is less than the resonance frequency, according to the theory of mixed liquid, we can get the sound velocity through the resonance of the water and air and the density. We can suppose $k, k_{a}, k_{w}$ to represent the compressibility of mixed liquid, air, and water separately, and presume $\rho, \rho_{\mathrm{a}}, \rho_{\mathrm{w}}$ are the corresponding density, when $\beta$ (the proportion of the air) in the water is very little, because the $k_{a} \gg k_{w}, \rho_{a} \ll \rho_{w}$, and $\beta \ll 1$, we can get the velocity:

$$
\begin{aligned}
v & =\left(\frac{1}{\rho k}\right)^{1 / 2} \\
& =\left\{\frac{1}{\left[\beta \rho_{a}+(1-\beta) \rho_{w}\right]\left[\beta k_{a}+(1-\beta) k_{w}\right]}\right\}^{1 / 2} \\
{[5 p t] } & =\left[\frac{1}{\rho_{w} k_{w}\left(1+\beta k_{a} / k_{w}\right)}\right]^{1 / 2}=v_{0}\left[\frac{1}{1+\beta k_{a} / k_{w}}\right]^{1 / 2} \\
& =\nu_{0}\left(\frac{1}{1+2.5 \times 10^{4} \beta}\right)^{1 / 2}
\end{aligned}
$$




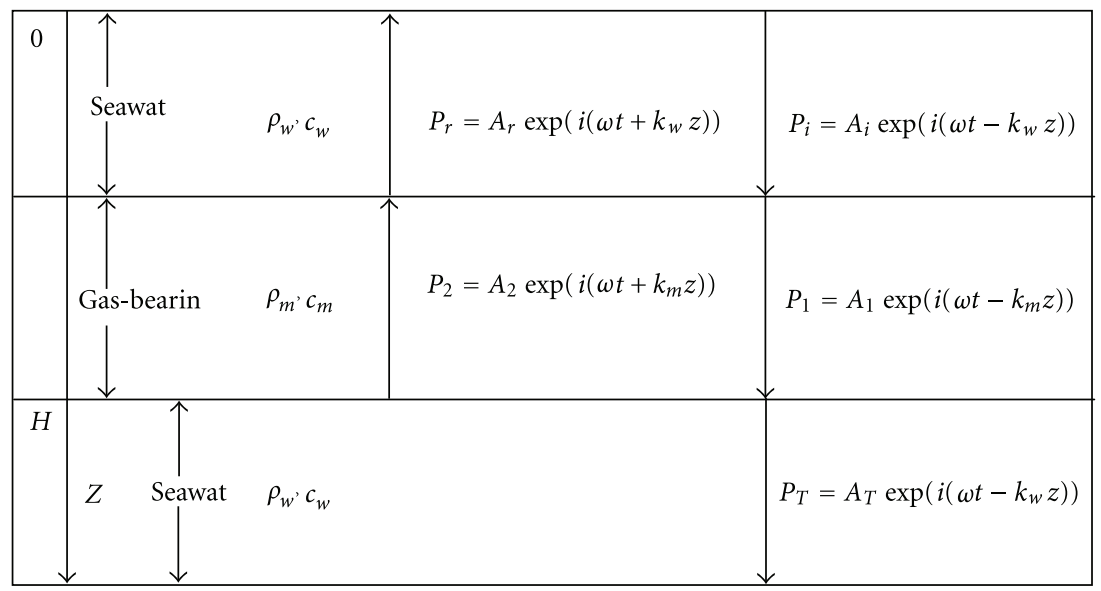

FIGURE 10: Reflection and transmission of sound wave in gas reservoir.

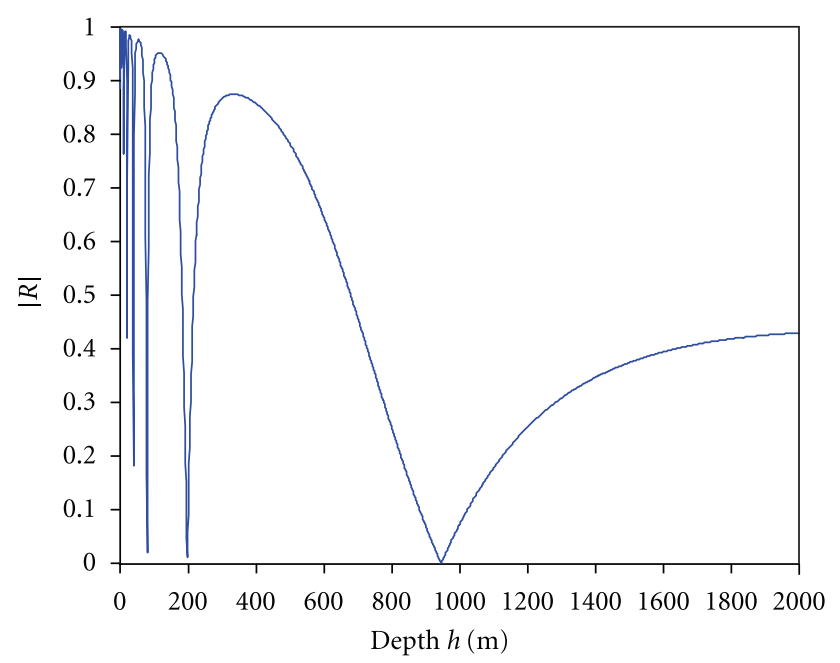

Figure 11: Curve of $|R|$ changed with depth $h$.

In (11), $v_{0}$ is the sound velocity of water without air. We can get that when the ratio of the gas in the mixed liquid is 0.01 , the velocity is about the $53.5 \%$ of that without air. Supposing the velocity is $\nu_{0}=1500 \mathrm{~m} / \mathrm{s}$ when there is no air, the curve is shown in Figure 7.

Conversely, when the frequency is higher than the resonance frequency of the mixed liquid with least air, which means that the frequency is higher than all the resonance frequency of the air, the influence of suspension gas can be ignored.

If the single bubbles distributing inside an area changed to be uniform, supposing the starting volume ratio is $0.01 \%$, the sound velocity varies following the depth, as in Figure 8. Through calculating, near the sea level, the velocity of sound in the layer is only $5 \%$ of that without gas.

When the frequency of sound approximately equals the air frequency, the velocity of sound wave has relations with not only the gas content but also the frequency of sound wave. If the frequency is approaching the resonance frequency, the speed of sound has a big change. Figure 9 is the smoothing speed curve of a group of homogeneous gas drawn by Fox et al. [2] under the conditions as these, the average diameter is $0.011 \mathrm{~cm}$, and the density $V_{a}=2 \times 10^{-4}$. When the frequency is resonant frequency, the speed of sound is the same as the velocity without air.

From Figure 9 we can see that when the frequency of sound wave is less than the resonant frequency of gas, the sound speed decreases because of the air; conversely, when the frequency of sound wave is higher than the resonant frequency of gas, the gas has little infection on the sound velocity; when the frequency of sound wave is approaching to the resonant frequency of gas, the sound velocity changes largely as the change of frequency.

When the frequency of the sound wave is almost the same as the resonant frequency of gas, and the diameter of sphere bubble distributed evenly far less than the wave length of sound, the accurate speed $c$ and reduction $\alpha$ under the effects of gas resonance is expressed as

$$
\begin{gathered}
\left(\frac{c_{0}}{c}\right)^{2}=\frac{1+A X}{2}\left\{1 \pm\left[1+\left(\frac{A Y}{1+A X}\right)^{2}\right]^{1 / 2}\right\}, \\
\alpha=\frac{c \pi f A Y}{c_{0}^{2}} .
\end{gathered}
$$

In the equation, $A=\rho c_{0}^{2} / p_{0} \gamma, \gamma$ is the adiabatic exponent, $c_{0}$ is the speed of water without bubble, $p_{0}$ is the pressure of static water, $\rho$ depends the density of gas and water mixture, and the parameters $X$ and $Y$ are presented as

$$
X=\frac{\beta\left(1-f_{*}^{2}\right)}{\left(1-f_{*}^{2}\right)^{2}+\delta_{*}^{2}}, \quad Y=\frac{\beta \delta_{*}}{\left(1-f_{*}^{2}\right)^{2}+\delta_{*}^{2}}
$$

Among (13), $f_{*}=f / f_{0}, \delta_{*}=\delta f_{*}^{2} \cdot f$ is the frequency of the sound wave, $f_{0}$ is the resonant frequency of gas bubble, and the $\delta$ is the damping constant.

2.3. Reflection and Transmission of Sound Wave. When the distance between the gases is less than the sound wavelength, the gas medium can be regarded as homogeneous medium, 


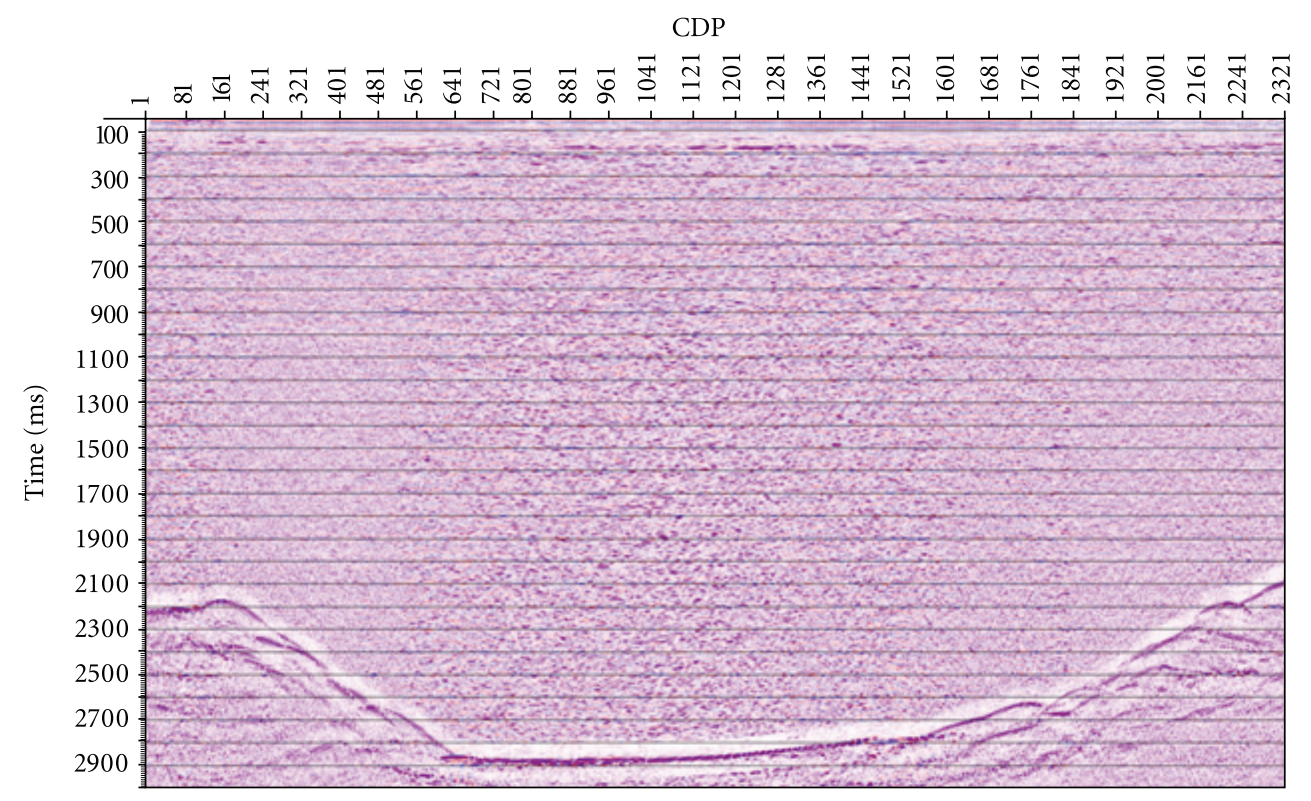

FIGURE 12: Characteristics of water bodies which appear in the high-resolution seismic profile of gas hydrate leakage area in the South China Sea.

because the gas in the sea water changes its compressibility, making the average density and velocity changed, the interface between gas-bearing water and seawater is the wave impedance interface. Therefore, the gas reservoir can be seen as an intermediate of the sea water medium at this time. If the wavelength is much longer than the radius of the gas $(r \ll \lambda / 2 \pi)$, suppose that a plane wave normally incident to a gas reservoir with the thickness of $H$, and the gas in it has the uniform size and uniform distributed (Figure 10), then the incident sound pressure is $P_{i}=A_{i} \exp (i(\omega t-$ $\left.k_{w} z\right)$ ), where $A_{i}$ is amplitude of the incident sound pressure; $P_{r}=A_{r} \exp \left(i\left(\omega t+k_{w} z\right)\right)$ is the reflection sound pressure, where $A_{r}$ is amplitude of the refection sound pressure; the transmission wave sound pressure is $P_{T}=A_{T} \exp (i(\omega t-$ $\left.k_{w} z\right)$ ), where $A_{T}$ is amplitude of the sound pressure; the layer transmission wave sound pressure is $P_{1}=A_{1} \exp (i(\omega t-$ $\left.k_{m} z\right)$ ), where $A_{1}$ is amplitude of the sound pressure; the reflection sound pressure is $P_{2}=A_{2} \exp \left(i\left(\omega t+k_{m} z\right)\right)$, where $\omega$ is the sound harmonic vibration circular frequency, $\rho_{w}$ and $\rho_{m}$ are the density of sea water and gasbearing, and $c_{w}$ and $c_{m}$ are the sound speed of sea water and gas-bearing, and $k_{w}=\omega / c_{w}$ and $k_{m}=\omega / c_{m}$ are the sound wave number of sea water and gas-bearing. The plane wave particle velocity is $v=v_{0} \exp (\omega t-k x)$, and the velocity amplitude is $v_{0}=p_{0} / c \rho$. Let $Z=c \rho$, then $Z_{w}$ and $Z_{m}$ are wave impedances of sea water and gas-bearing.

The velocity and pressure on interface between sea water and gasbearing are continuous, so the boundary conditions are as follows

(1) when $z=0$, pressure: $P_{i}+P_{r}=P_{1}+P_{2}$, that is,

$$
A_{i}+A_{r}=A_{1}+A_{2}
$$

velocity: $\left(P_{i}-P_{r}\right) / Z_{w}=\left(P_{1}-P_{2}\right) / Z_{m}$, that is,

$$
\frac{A_{i}-A_{r}}{Z_{w}}=\frac{A_{1}-A_{2}}{Z_{m}}
$$

(2) when $z=H$, pressure: $P_{1}+P_{2}=P_{T}$, that is,

$$
A_{1} \exp \left(-i k_{m} H\right)+A_{2}\left(i k_{m} H\right)=A_{T} \exp \left(-i k_{w} H\right)
$$

velocity: $\left(P_{1}-P_{2}\right) / Z_{m}=P_{T 1} / Z_{W}$, that is,

$$
\frac{A_{1} \exp \left(-i k_{m} H\right)-A_{2} \exp \left(i k_{m} H\right)}{Z_{m}}=\frac{A_{T} \exp \left(-i k_{w} H\right)}{Z_{W}} .
$$

Simultaneous equations (14), (15), (16), and (17), and making $R=A_{r} / A_{i}, T=A_{T} / A_{i}, B_{1}=A_{1} / A_{i}, B_{2}=A_{2} / A_{i}$, then

$$
\begin{aligned}
R & =\frac{\left(Z_{w}^{2}-Z_{m}^{2}\right)\left[1-\exp \left(i 2 k_{m} H\right)\right]}{\left(Z_{m}+Z_{w}\right)^{2} \exp \left(i 2 k_{m} H\right)-\left(Z_{w}-Z_{m}\right)^{2}}, \\
T & =\frac{4 Z_{m} Z_{w} \exp \left[i\left(k_{m}+k_{w}\right) H\right]}{\left(Z_{m}+Z_{w}\right)^{2} \exp \left(i 2 k_{m} H\right)-\left(Z_{m}-Z_{w}\right)^{2}}, \\
B_{1} & =\frac{2 Z_{m}\left(Z_{m}+Z_{w}\right) \exp \left(i 2 k_{m} H\right)}{\left(Z_{m}+Z_{w}\right)^{2} \exp \left(i 2 k_{m} H\right)-\left(Z_{m}-Z_{w}\right)^{2}}, \\
B_{2} & =\frac{2 Z_{m}\left(Z_{w}-Z_{m}\right)}{\left(Z_{m}+Z_{w}\right)^{2} \exp \left(i 2 k_{m} H\right)-\left(Z_{m}-Z_{w}\right)^{2}} .
\end{aligned}
$$




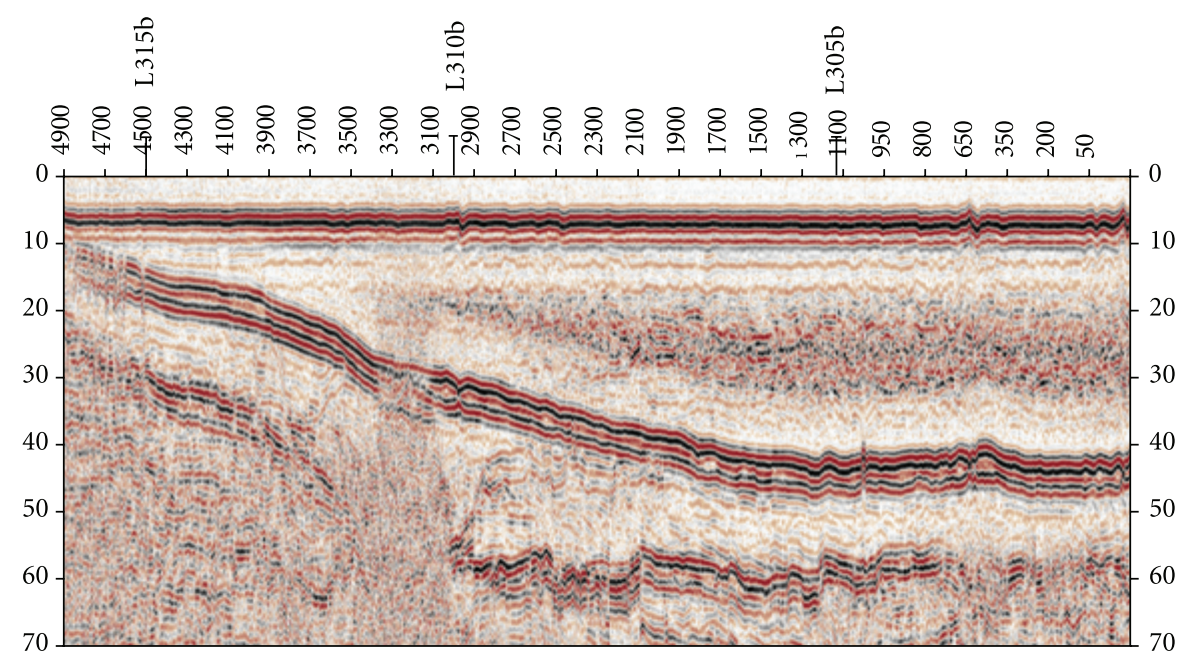

FIGURE 13: Characteristics of water bodies which appear in the high-resolution seismic profile of shallow gas hydrate leakage area in the Bohai Sea.

The particle displacement is $\xi=\left(v_{0} / i \omega\right) \exp (i(\omega t-k x))$ for plane wave, and the displacement amplitude is $\xi_{0}=v_{0} / i \omega$, at the point of $x=0, R=A_{r} / A_{i}=v_{r 0} / v_{i 0}=\xi_{r 0} / \xi_{i 0}$ is the ratio of the displacement amplitude of incident and reflected waves on the interface. Similarly, $T$ is the ratio of the amplitude of incident and wave transmission through the gas, $B_{1}$ is the ratio of the amplitude of incident and wave transmission of the above interface, $B_{2}$ is the ratio of the amplitude of incident and wave transmission of the following interface.

Assuming a floating gas layer, in which the size of the gas is uniform distribution, with the thickness of $H=0.01 \mathrm{~m}$ remaining unchanged, the size of the gas at the depth of $2000 \mathrm{~m}$ is the size of gas assumed in this paper. The volume ratio is $\beta=0.01 \%$. When a plane wave with the frequency of $30 \mathrm{kHz}$ normal incident, the amplitude ratio $R$ of incident and reflection is shown as Figure 11.

As can be seen from Figure 11, $|R|$ has certain cyclical changes. When $k_{m} H=n \pi(n=0,1,2, \ldots)$, the thickness of the bubble layer is an integer multiple of half wavelength, then $|R|$ is minimum, for example, at the point of $h=947 \mathrm{~m}$, the wave is almost the transmission sound. When $k_{m} H=$ $(2 n-1) \pi / 2$, the thickness of the gas layer is an odd multiple of the quarter wavelength, then $|R|$ is maximum, for example, at the point of $h=338 \mathrm{~m}$, almost all the sound wave reflects back.

2.4. Scattering Effect of Natural Gas. The attenuation yielded by sound absorption needs to be considered in the actual gas. While the gas is forced to vibrate, some sound energy is burned because of the system's internal friction and distributed to the surrounding water in the form of heat, but the large vibration attenuation prevented the temperature balance in every moment. When the gas is compressed, the heat is diffused along with the increasing temperature, so the bubble began to cool prior to the expansion. When the volume of gas is compressed to a minimum, the temperature is slightly reduced because of the heat shed to the water. The same is true when the expansion. Therefore, the process carried out in the gas is the procession between adiabatic process and isothermal process. Taking all these circumstances into account, the scattering cross-section expression of real gas is

$$
\sigma_{s}=\frac{4 \pi r^{2}}{\left(f_{0}^{2} / f^{2}-1\right)^{2}+\delta^{2}}
$$

in (19), $\delta$ is total damping value, of which expression is

$$
\delta=\frac{f_{0}^{2}}{f^{2}} \beta+\eta+\frac{C_{1}}{c \rho \eta}
$$

in (20), $\beta$ is normal number; $\beta \ll 1, C_{1}$ is constant, to represent friction effect.

From (19), can obtain the ration of scattering crosssection and geometric cross section:

$$
\frac{\sigma_{s}}{\pi r^{2}}=\frac{4}{\left(f_{0}^{2} / f^{2}-1\right)^{2}+\delta^{2}} .
$$

When the sound frequency is closed to resonance frequency, $\sigma_{s 0} / \pi r^{2}=4 / \delta_{0}^{2}, \delta_{0}$ is called the total damping constant.

For the real gas, the absorption of sound energy must be considered. The sum of scattering energy and absorption energy is called dissipation energy while the dissipation section is

$$
\sigma_{e}=\sigma_{s}+\sigma_{a}=\frac{4 \pi r^{2}(\delta / \eta)}{\left(f_{0}^{2} / f^{2}-1\right)^{2}+\delta^{2}}
$$

So the absorption cross-section can be calculated as

$$
\sigma_{a}=\frac{4 \pi r^{2}(\delta / \eta-1)}{\left(f_{0}^{2} / f^{2}-1\right)^{2}+\delta^{2}} .
$$


It can be seen that the relationship of dissipation section and scattering cross-section is $\sigma_{e} / \sigma_{s}=\delta / \eta$.

\section{Effect of Actual Data}

Deep -ea high-resolution multichannel seismograph system developed by Ocean University of China was used in March 2009. It is an all new digital marine seismic hydrophone and the most advanced new marine seismic hydrophone all-digital instrument system in the world. Compared with the current analog seismic hydrophone system, its domestic applicability is stronger, and its resolution and SNR are higher. Each hydrophone has 24 channels and can be combined into a system of $24 \sim 1940$ channels. The diameter of hydrophone is only $38 \mathrm{~mm}$ and the wall thickness is $1 / 8$ inch. That is easy for transport and delivery and is durable. Using $6.25 \mathrm{~m}$ group interval and $1 / 16 \sim 4 \mathrm{~ms}$ broadband technology, it can be applied in oil exploration, marine engineering, and stratigraphic section measurement to obtain high-resolution seismic data. The high-resolution seismic data acquisition was firstly used in gas hydrates area near Dongsha Islands in the South China Sea. Record length of survey line is $3 \mathrm{~s}$, the sampling interval is $1 \mathrm{~ms}$, and the number of coverage is 6 times.

For feature of study areas that have a wide range of leakage area (including hydrate and free gas) in favor gas hydrate $[12,13]$, various gas hydrate leakage areas (including gas hydrate and free gas) distributions have been found through the geological survey studies. With the recent increase in understanding and investigating instrument performance improvement, we can further understand the distribution of gas hydrate leakage area (including gas hydrate and free gas) and seismic character (Figure 12), through the study on gas hydrate leakage area (including hydrate and free gas) according to seismic survey data.

For further research on the seismic character in shallow gas leakage area (including hydrate and free gas), according to the research and experimental observation of water body features (Figure 13) on high-resolution seismic profile in the Bohai Sea shallow gas leakage area, the following sound properties of sediments and water that contains gas have been found. (1) The nature of the sound parameters of the gas. (2) The sound characteristics in the water contains natural gas (gas in shallow water, and the vibration process, resonance frequency, sound velocity, attenuation, reflection, and transmission). (3) The sound characteristics of sediments contain natural gas (resonant frequency, bubble damping, sound velocity, and attenuation).

In recent years seismic reflection characteristics of gas hydrates in deep water (Figure 12) and shallow gas in shallow water (Figure 13) leakage area (including hydrate and free gas) were summarized. Thus the seismic characteristics of the natural gas leakage area (containing gas hydrate and free gas) is divided into three categories according to spatial location. (1) The seismic characteristics in the formation: sound blanket, sound curtains, sound disturbances, irregular strong reflecting surface interface, and phase-down on both sides. (2) Features in the seabed surface: submarine pockmark and large collapse pit. (3) Features in water layer: sound plumes, cloud-like move, and dash-dot reflection. Combination of theory and case analysis shows that (1) there exists gas escaped from buried natural gas area in the sound plumes, cloud-like disturbance, and dash-dot reflection areas in seawater; (2) the region of sound blanket space represents areas of high gas concentration, a variety of sound disturbances represents areas with low gas concentration, and the gas concentration in sound curtains between the front two conditions.

\section{Conclusions}

The following conclusions can be obtained by the study of features of bubbly water in gas leakage area (including hydrate and free gas) through the study of characteristics of elastic parameters of deposition in the gas leakage area (including hydrate and free gas), and the use of sound theory of water and sediment containing hydrate and free gas.

(1) We get the changes of the volume and radius when the gas rises at different depths, and also get the expression of acceleration in the static water. Besides, since the dynamic viscosity becomes smaller when the gas rises, the force gas suffered and the velocity keeps increasing until it arrives at the surface. Radius and volume of gas changes with depth with different velocities, in the context of $400 \mathrm{~m}$ below sea level, the velocity changes very fast, and as the depth increases the changes become smaller.

(2) We also give the expression of reflection coefficient of gas, the size of the reflection coefficient not only related to impedance of both sides of the interface, but also related to the product of wave number and thickness of the gas, the reflection coefficient shows a certain periodicity. The gas becomes bigger when rising, volume ratio grows and sound is attenuated faster, Theoretically, there are only $5 \%$ of no gas medium reaching sea level.

(3) The size of the cross-section represents the gas range of the sound scattering, When the pressure is constant, scattering cross-section reaches the maximum near the resonance frequency, these conditions are changing as the gas rises, and the maximum of the scattering cross-section is only one-tenth of its geometric cross-section. Sound energy endure losses through internal friction and thermal conduction when the actual gas vibrates. This loss of performance for the scattering and absorption of air bubbles, absorption cross-section is less than the scattering cross section.

(4) The main sound features of gas are relevant to gas size, gas content, velocity, attenuation, resonance frequency and the scattering cross-section, and other conditions. We can clearly see the sound characteristics of seismic data that yielded when the gas (or gas hydrate dissociation) escaped the water. 


\section{Acknowledgments}

This paper is supported by the National Basic Research Program (973) subjects (2009CB219505) and Natural Science Foundation of Shandong Province (ZR2010DM012).

\section{References}

[1] E. L. Carstensen and L. L. Foldy, "Propagation of sound through a liquid containing bubbles," Journal of Acoustical Society of America, vol. 19, no. 3, pp. 481-501, 1947.

[2] F. E. Fox, S. R. Curley, and G. S. Larson, "Phase velocity and absorption measurements in water containing bubbles," Journal of the Soundal Society of America, vol. 27, no. 3, p. 534, 1955.

[3] E. Silberman, "Sound velocity and attenuation in bubbly mixtures measured in standing wave tubes," Journal of Acoustical Society of America, vol. 29, no. 8, pp. 925-933, 1957.

[4] Z. Xiaoliang, Z. Zhemin, and Z. Lin, "Analytical description of sound wave propagation in a bubbly liquid and its strong nonlinearity," Applied Sounds, vol. 18, no. 6, pp. 18-23, 1999.

[5] A. Prosperetti, "Thermal effects and damping mechanisms in the forced radial oscillations of gas bubbles in liquids," Journal of Acoustical Society of America, vol. 61, no. 1, pp. 17-27, 1977.

[6] M. Kameda and Y. Matsumoto, "Nonlinear oscillation of a spherical gas bubble in acoustic fields," Journal of the Acoustical Society of America, vol. 106, no. 6, pp. 3156-3166, 1999.

[7] K. W. Commander and A. Prosperetti, "Linear pressure waves in bubbly liquids: comparison between theory experiments ," Journal of the Acoustical Society of America, vol. 85, no. 2, pp. 732-746, 1989.

[8] A. Prosperetti, L. A. Crum, and K. W. Commander, "Nonlinear bubble dynamics," Journal of the Acoustical Society of America, vol. 83, no. 2, pp. 502-514, 1988.

[9] F. Li, J. Sun, and J. Huang, "Study on propagation of sound wave in a bubbly liquid," Journal of Northwestern Polytechnical University, vol. 16, no. 2, pp. 241-245, 1998.

[10] G. Yonghui, "Concentration of entrained air in air-water mixture as measured by sound transmission loss," Applied Sounds, vol. 18, no. 1, pp. 32-35, 1999.

[11] S. Vagle and M. F. David, "The measurement of bubble-size distribution by soundal backscatter ,"Journal of Atmos-Pheric and Oceanic Technology, vol. 9, pp. 630-644, 1992.

[12] L. Huaishan and Z. Zhengyun, "Seismic data processing approaches for the study of gas hydrates in the East China Sea," Journal of Ocean University of Qingdao, vol. 1, no. 1, pp. 87-92, 2002.

[13] H. Liu, G. Huang, Y. He, S. Tong, S. Cui, and J. Zhang, "AVO character research of natural gas hydrates in the East China Sea," Journal of Ocean University of Qingdao, vol. 8, no. 3, pp. 270-276, 2009. 

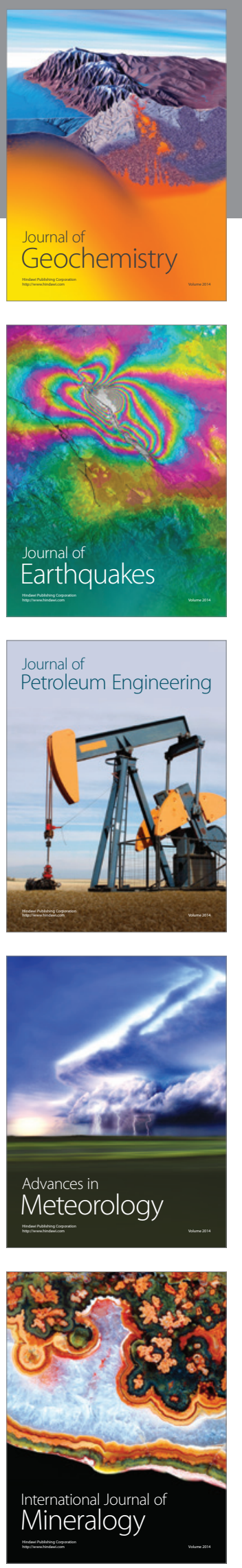
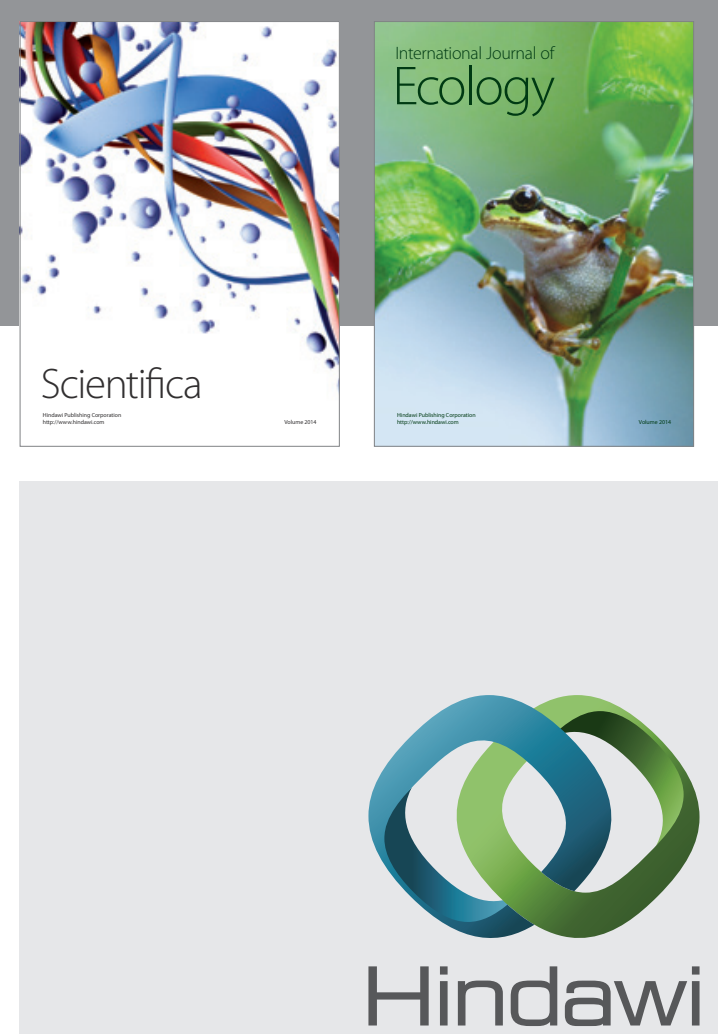

Submit your manuscripts at http://www.hindawi.com
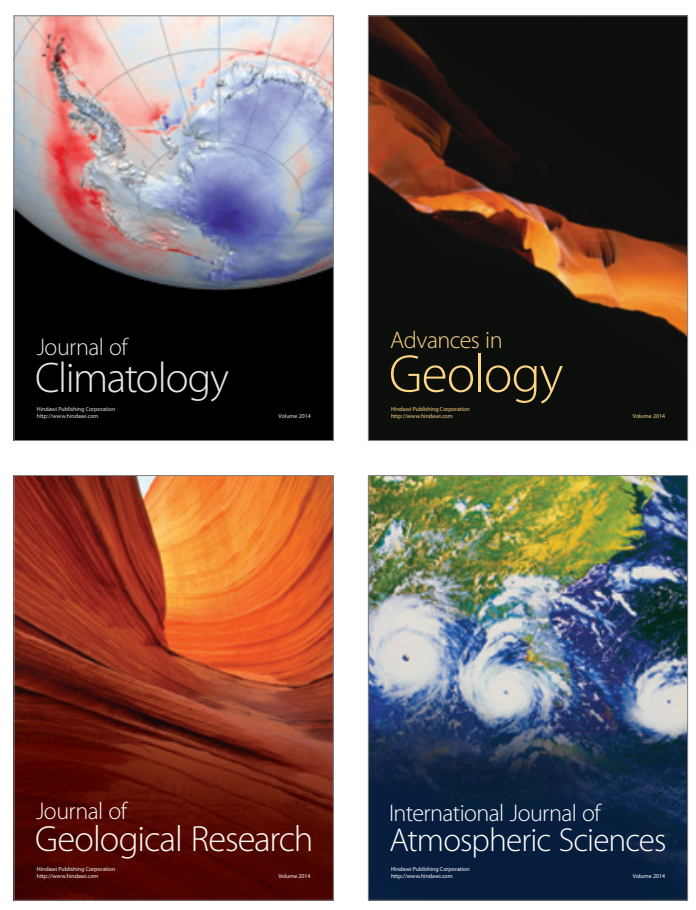
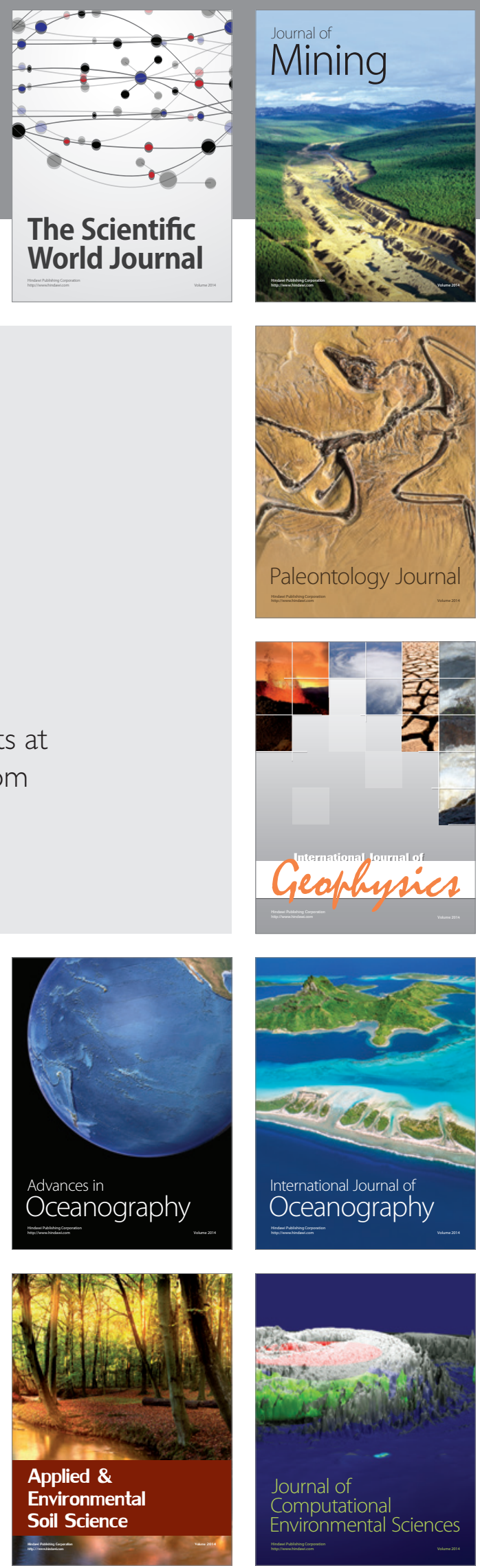